\title{
Siemens Betriebskrankenkasse unterstützt KHK-Prävention Gesundheitsbewusstein kommt oft erst mit dem Infarkt
}

\author{
Einen Noch-Gesunden mit KHK- \\ Risikofaktoren zur Prävention zu mo- \\ tivieren, ist in der Regel schwieriger, \\ als einen Postinfarktpatienten dafür \\ zu gewinnen. Die Krankenkassen \\ können dabei Unterstützung bie- \\ ten, wie das Versorgungsangebot \\ „KardioPro“ der Siemens Betriebs- \\ krankenkasse (SBK) belegt.
}

- Trotz gewisser Fortschritte ist der Myokardinfarkt immer noch eine der häufigsten Todesursachen. „Dabei ist der Herzinfarkt kein schicksalhaftes Ereignis, sondern das Ergebnis eines vielfältigen Fehlverhaltens - wobei jeder einzelne Fehler zunächst ohne Folgen zu sein scheint", sagte Walter Haßelbacher, Fachgebietsleiter der SBK. Die entscheidenden Risikofaktoren für die KHK sind neben Hypertonie und Diabetes vor allem Bewegungsmangel, Übergewicht, Stress und Rauchen.

Der Praxisalltag zeigt, dass Herzkranke ihre Risikofaktoren im Allgemeinen besser im Griff haben als Noch-Gesunde. „Deutlich weniger engagiert im Sinne einer Gesundheitserhaltung sind Menschen, die zwar erhöhte Risiken zeigen, aber noch nicht krank sind", so Haßelbacher. Erst wenn sich die KHK manifestiert habe, entstehe die Bereitschaft, den Lebensstil zu ändern.

\section{Den inneren Schweinehund überwinden!}

Unbestritten spielen Ärzte beim Erkennen und der Korrektur der Risikofaktoren die zentrale Rolle. Doch können Krankenkassen die Bemühungen der Ärzte unterstützen. Zum Beispiel bietet die SBK ihren Versicherten den KardioPro Risikocheck an. „Mit diesem Test werden die entscheidenden Risikofaktoren erfasst und Patienten identifiziert, die trotz Beschwerdefreiheit bereits eine KHK haben“", so Haßelbacher.
Beim SBK KardioPro Risikocheck werden Blutdruck, Cholesterin und Blutzucker bestimmt, aber auch Rauchen und andere Risikofaktoren erfasst. Anhand des PROCAM-Scores lässt sich berechnen, wie hoch das Risiko ist, in den nächsten Jahren einen Herzinfarkt oder einen plötzlichen Herztod zu erleiden. Bei einem Wert $<1 \%$ besteht ein niedriges, bei Werten $>2 \%$ ein hohes KHK-Risiko, das neben lebensdiätetischen auch medikamentöse Therapiemaßnahmen erfordert.

Bei Patienten mit niedrigem Risiko empfiehlt sich die erneute Beurteilung des Infarktrisikos erst nach fünf Jahren. Bei Patienten mit mittlerem oder hohem Risiko werden zusätzliche Untersuchungen wie ein Belastungs-EKG oder eine Kalkscore-Bestimmung durchgeführt. Außerdem erhalten sie eine intensive Beratung und Unterstützung im Hinblick auf die Korrektur der Risikofaktoren Rauchen, Ernährung und Bewegung, nach dem Motto: Lust auf Ge- sundheit - den inneren Schweinehund überwinden!

\section{Erste Erfolge von KardioPro}

Erste Daten belegen, dass das KardioPro-Projekt durchaus erfolgreich ist. Von 10800 Teilnehmern fand sich bei $11 \%$ ein hohes und bei $18 \%$ ein mittleres KHK-Risiko. In der Hochrisikogruppe konnte durch die Präventionsmaßnahmen innerhalb eines Jahres z. B. das LDL-Cholesterin von durchschnittlich 156 auf $131 \mathrm{mg} / \mathrm{dl}$ gesenkt werden. Der Anteil der Raucher ging von 21 auf $15 \%$ zurück und der systolische Blutdruck sank von 146 auf $139 \mathrm{mmHg}$.

Die Versorgungsinitiative KardioPro wird extrabudgetär vergütet. Da das Projekt von einem Versorgungsnetz getragen wird, ist jedem Patienten auch eine sofortige Terminabsprache beim Facharzt bzw. in der Klinik garantiert.

Dr. med. Peter Stiefelhagen =

- SBK-Symposium im Rahmen der Tagung „Der herzkranke Diabetiker“, Berlin, 3.12.2010

\section{Prävention als gesellschaftspolitische Aufgabe}

\section{"Gesundheitskompetenz fördern - vom Kindergarten an"}

MMW: Glauben Sie, dass Maßnahmen wie das SBK-Projekt „KardioPro“ Herzinfarkte erfolgreicher verhindern können als der erhobene Zeigefinger des Arztes?

Tschöpe: Grundsätzlich ja, wenn sie eingebettet sind in ein sinnvolles und attraktives Kommunikationsprogramm, das es dem Versicherten leicht macht mitzuwirken - nach dem Motto: „Gesund zu bleiben bzw. zu sein macht Spaß!“

MMW: Was fordern Sie von der Politik, um einer effektiven Prävention zum Durchbruch zu verhelfen?

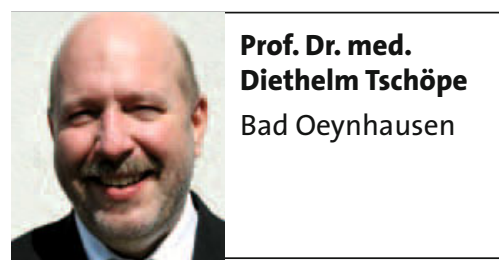

Tschöpe: Sie muss den Bürgern klar machen, dass Gesundheit ein individuelles und gesellschaftliches Gut ist. Das beinhaltet die Förderung von Gesundheitskompetenz vom Kindergarten bis ins Erwachsenenalter. Prävention ist mindestens genauso viel Gesellschaftspolitik wie Gesundheitspolitik. Es wird darauf ankommen, die richtigen gesellschaftlichen Anreize dafür zu setzen. 